\title{
OBSERVACIONES FENOLÓGICAS DE BLAKEA SCARLATINA (MELASTOMATACEAE) RESERVA BIOLÓGICA ALBERTO MANUEL BRENES, SAN RAMÓN, ALAJUELA, COSTA RICA ${ }^{1}$
}

\author{
PHENOLOGICAL OBSERVATIONS OF BLAKEA SCARLATINA \\ (MELASTOMATACEAE) ALBERTO MANUEL BRENES BIOLOGICAL \\ RESERVE, SAN RAMÓN, ALAJUELA, COSTA RICA
}

Liz Brenes Cambronero ${ }^{2}$

Ronald Sánchez Porras ${ }^{3}$

\begin{tabular}{|l|l} 
Recibido: 08.04.19 & Aprobado: 09.12.19
\end{tabular}

DOI: $10.15517 /$ isucr.v20i42.41854

\section{Resumen}

Se estudió las fenoetapas de Blakea scarlatina y su asociación con factores biofísicos, de marzo 2014 a octubre 2015. La muestra se seleccionó por orden de aparición. Las fenoetapas se evaluaron cada mes, con escala de 0 a cuatro. Brotes foliares y caída de follaje se presentaron durante el estudio, este último excepto en junio 2014. La floración se presentó en el 64\% de la investigación, esto hace a $B$. scarlatina una especie atractiva como ornamental. Se registraron diferencias intraespecíficas en las fenoetapas. La caída de follaje y brotes fueron inversamente proporcional con la temperatura. La floración se asoció con temperatura, caso inverso con humedad relativa. La fructificación se asoció con precipitación.

Palabras claves: Blakea scarlatina; arbusto epifito; fenología; fenoetapas; bosque tropical premontano; floración continua.

\footnotetext{
${ }^{2}$ Costarricense, Bióloga, Docente investigadora en la Universidad de Costa Rica, Sede de Occidente, Sección de Biología. Programa de Investigaciones en Gestión Sostenible de los Recursos Naturales. Correo electrónico llmbrenes@hotmail.com

${ }^{3}$ Costarricense, Biólogo, Docente investigador en la Universidad de Costa Rica, Sede de Occidente, Sección de Biología. Programa de Investigaciones en Gestión Sostenible de los Recursos Naturales. Correo electrónico ronald.rsr@gmail.com
} 


\begin{abstract}
:
The phenofases of Blakea scarlatina and its association with biophysical factors were studied, from March 2014 to October 2015. The sample was selected in order of appearance. The phenofases were evaluated every month, with a scale of 0 to four. Budding and leaf abscission occurred during the study, the last one except in June 2014. Flowering occurred in 64\% of the research, this makes $B$. scarlatina an attractive ornamental species. Intraspecific differences were recorded in the phenofases. The leaf abscission and budding were inversely proportional to the temperature. Flowering was associated with temperature, inverse case with relative humidity. Fruiting was associated with precipitation.
\end{abstract}

Key words: Blakea scarlatina; epiphytic shrub; phenology; phenofases; premontane tropical forest; continuous flowering.

La familia Melastomatacea tiene cerca de 170 géneros y de 4200 a 4500 especies con una distribución pantropical con una concentración acentuada en el neotrópico en donde ocurren cerca de 3000 especies (Martins, 2009). En Costa Rica, esta familia se encuentra muy bien representada en hierbas, árboles, arbustos o bejucos, se pueden localizar de 0-3330 msnm (Azofeifa-Rojas, 2015).

Asimismo, estos autores mencionan que las especies arbustivas de Melastomataceae son muy atractivas como ornamentales por la cantidad y belleza de las flores, que pueden variar de blanco, rosado, morado o rojo. Las flores son visitadas por abejas y muchos otros insectos. Los frutos de colores brillantes, son muy apreciados por aves (Azofeifa-Rojas, 2015).

Uno de los géneros de las Melastomataceae es Blakea, el cual tiene aproximadamente 100 especies, con frutos tipo baya y está mejor representado en regiones montañosas húmedas del sur de México y las Antillas hasta Bolivia y Brasil. El noroeste de América del Sur es el centro de riqueza de este género. En tanto que el sur de Centroamérica es el área de importancia para la diversidad inusual en morfología floral (Almeda 2000).

Blakea scarlatina Almeda es un arbusto epífito que tiene como características principales sus flores conspicuas y abundantes, sumamente atractivas, ya que sus pétalos son color escarlata y contrasta con el amarillo intenso de sus anteras. Almeda (1990 y 2000) menciona que las flores de esta especie son solitarias con anteras oblongas, 7-8 mm de largo y 4-5 mm de grueso de color amarillo, las brácteas florales son anchas con pétalos rojos brillantes y con anteras conectivas prominentes. Se ha observado flores en diferentes momentos del año. 
La distribución geográfica de B. scarlatina según Almeda (2000) es localizada y poco común (rara). La mayoría de los individuos se encuentran en las tierras bajas del Caribe o las vertientes de Nicaragua y Costa Rica en el bosque lluvioso y nuboso, a una altitud de 0-1450 msnm. En el área de estudio contiguo a la Reserva Biológica Alberto Manuel Brenes se puede observar está Blakea a una altitud de 767 a 861 msnm.

Dado el potencial que la especie en estudio tiene, se hace necesario estudiar su biología reproductiva y fenología. De acuerdo con Boyle y Bronstein (2012) la información sobre la descripción básica de los patrones fenológicos tropicales y de los procesos que los afectan es muy escasa. La fenología puede entenderse como el estudio de las respuestas de los organismos vivos a los diferentes cambios y estímulos originados por el medio externo, principalmente el clima y las interacciones con las otras formas de vida (Pérez, Villalba y Almanza, 2013). La fenología también comprende el estudio de los eventos biológicos cualificables o cuantificables que ocurren cíclicamente a lo largo de la vida de las plantas, los cuales están determinados por un conjunto de factores climáticos, edáficos y bióticos (Borchert 1983, Gómez-Restrepo 2010-2011, Schwartz 2003).

Estos cambios estacionales, pueden generar fluctuaciones en la frecuencia y abundancia de polinizadores, agentes dispersores de semillas, depredadores y competidores (Lieberman 1982). A nivel intraespecífico, la sincronización y la duración de los eventos fenológicos son características importantes que influyen en las interacciones de las plantas con sus dispersores, herbívoros o polinizadores (Rathcke y Lacey, 1985; Primack,1987).

Por lo anterior, esta investigación tuvo como objetivo observar la fenología de $B$. scarlatina en cuanto a sus fenofases en caída de follaje, aparición de brotes foliares, floración y fructificación, durante 20 meses, así como el grado de asociación de las fenofases con la humedad relativa, precipitación y temperatura ambiental.

Materiales y métodos

La presente investigación se llevó a cabo desde marzo del 2014 hasta octubre 2015, a ambos lados del camino lastrado $(13 \mathrm{~km})$ que conduce y se encuentra dentro de la Reserva Biológica Alberto Manuel Brenes (ReBAMB). La Reserva se ubica en la parte sur de la Cordillera de Tilarán, 
Costa Rica (coordenadas 0439494E y $1131058 \mathrm{~N}$ ), tiene una precipitación promedio de 3500mm, temperatura promedio $21^{\circ} \mathrm{C}$ y la zona de vida bosque pluvial premontano (Brenes, 1999).

En un recorrido de seis $\mathrm{km}$ desde el camino de entrada a la ReBAMB hasta $50 \mathrm{~m}$ después del portón de ingreso se ubicó, por orden de aparición una muestra de 12 individuos de B. scarlatina (Melastomataceae) (L. Brenes Cambronero 60 (USJ)) con diámetro a la altura del pecho (DAP) superior o igual a $11 \mathrm{~cm}$ y una altura promedio de 9,2 m (Cuadro 1). Para identificar todos los individuos, se les colocaron marcas plásticas enumeradas y se georreferenciaron en coordenadas CRTM05 para facilitar su ubicación y posteriores observaciones. El primer árbol se ubicó en las coordenas 0437758 Oeste y 1131545 Norte y el último individuo en las coordenadas 0436669 Oeste y 1129988 Norte.

Cuadro 1. Altura y diámetro de los ejes observados de B. scarlatina (n=8), ReBAMB, Costa Rica. Marzo 2014 a junio 2015.

Table 1. Height and diameter of the axes observed of B. scarlatina $(\mathrm{n}=8)$, ReBAMB, Costa Rica. March 2014 to June 2015.

\begin{tabular}{|c|c|c|}
\hline No. individuo & Altura $(\mathrm{m})$ & Diámetro $(\mathrm{cm})$ \\
\hline 1 & 9 & - \\
2 & - & - \\
3 & 8 & 12 \\
4 & 11 & 12 \\
5 & 15 & 10 \\
6 & 2,5 & 9 \\
7 & - & - \\
8 & 8 & - \\
9 & - & - \\
10 & 10 & 12 \\
11 & - & - \\
12 & - & - \\
\hline Media & 9,2 & 11 \\
\hline
\end{tabular}

Nota: este cuadro incluye aquellas plantas en las que fue posible hacer las mediciones correspondientes.

A cada individuo se le evaluó las siguientes etapas fenólogicas: caída de follaje (cf), aparición de brotes foliares (br), floración (fl) y fructificación (fr) (Figura 1). De acuerdo con Almeda (1990) describen que este arbusto epífito se caracteriza por tener de 2-5m de altura, hojas 
elípticas a elíptico ovadas de 10-18cm de largo y 6-8 cm de ancho. Además, las hojas se distinguen por ser triplinervias con nervios secundarios transversales mayormente a 1-2 mm de distancia. Las flores son solitarias con anteras oblongas, 7-8 $\mathrm{mm}$ de largo y 4-5 $\mathrm{mm}$ de grueso de color amarillo. Los frutos son bayas.

Las observaciones se realizaron con el uso de binoculares y desde los mejores ángulos posibles. Las observaciones fenológicas se realizaron una vez por mes y con escala de 0 a cuatro; donde 0 es ausencia de la etapa fenológica, 1 corresponde desde 1\% hasta 25\%, 2 comprende desde $26 \%$ hasta $50 \%$, 3 desde $51 \%$ a $75 \%$ y 4 de $76 \%$ a $100 \%$ de presencia en la fenoetapa (Fournier, 1974).

Se utilizaron correlaciones lineales calculadas con los datos promedios de 10 años de la estación meteorológica de la ReBAMB. La humedad relativa, precipitación y temperatura se correlacionaron con caída de follaje, aparición de brotes foliares, floración y fructificación.

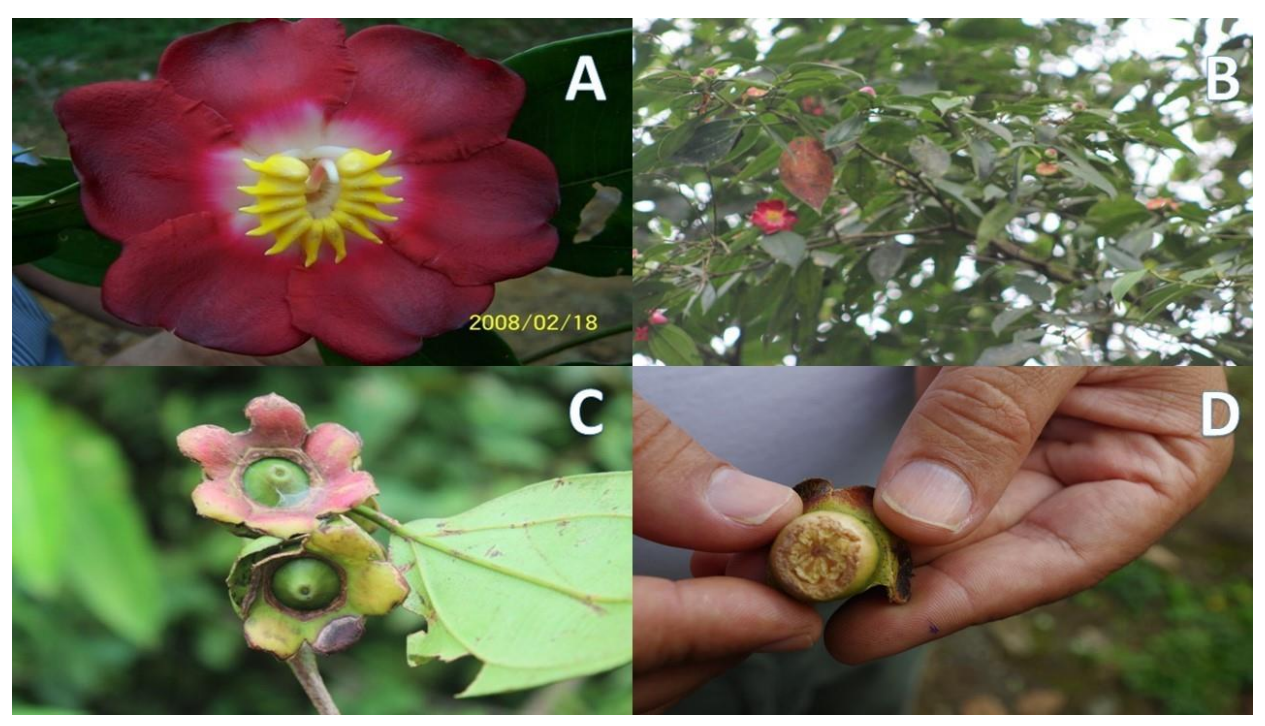

Figura 1. Floración (A), flores y frutos (B), frutos (C) y frutos (D) de B. scarlatina (n=8). Imágenes tomadas de marzo 2014 a octubre 2015. Reserva Biológica Alberto Manuel Brenes, Costa Rica.

Figure 1. Flowering (A), flowers and fruits (B), fruits (C) and fruits (D) of B. scarlatina $(\mathrm{n}=8)$. Images taken from March 2014 to October 2015. Alberto Manuel Brenes Biological Reserve, Costa Rica. 
Resultados

Los ocho individuos de $B$. scarlatina observados en el estudio fenológico, se ubicaron entre los 760 hasta los 860 msnm en un bosque pluvial premontano. Estos individuos presentaron caída de follaje durante todo el estudio, excepto en junio 2014, en la que este fenómeno no ocurrió. El máximo valor fenológico en este aspecto fue de 2,7 que se registró en el mes de febrero 2015 (Figura 2).

En cuanto a la presencia de brotes foliares se encontraron durante todo el estudio. Los valores más bajos de brotes foliares, fueron cercanos a uno, en los meses de marzo y agosto 2014 y junio 2015 (Figura 2).

B. scarlatina produjo flores en nueve de los 14 meses de investigación (Figura 2). En el promedio de la muestra estudiada, se observa en el mes de agosto 2014 el valor más alto de floración $(2,3)$.

Se observó en la media de la población que el proceso de producción de frutos, tuvo una máxima de tres en setiembre 2014 y otros dos picos de 1,3 en abril 2014 y marzo 2015. Solo durante cuatro meses (agosto 2014-noviembre 2014) hubo una tendencia que en cuanto a mayor aparición de brotes también existió mayor cantidad de frutos. También se observó que hay un traslape en las fenofases de floración y fructificación en tres momentos del estudio (Figura 2).

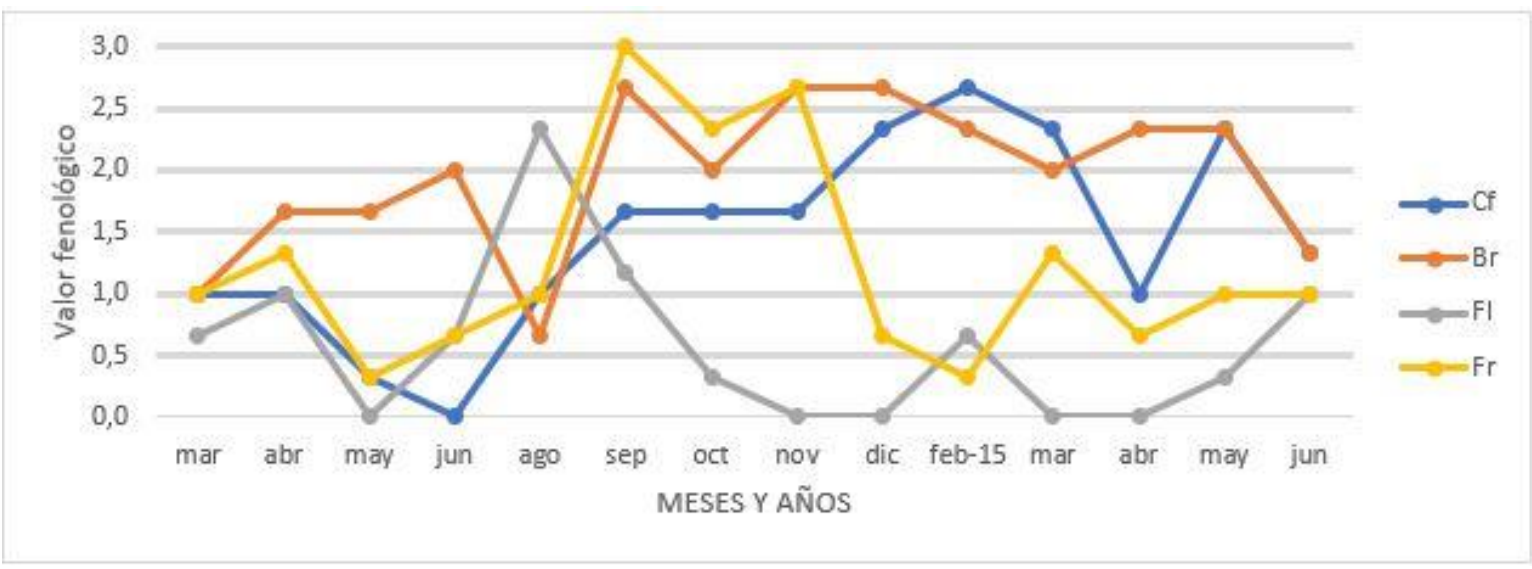

Figura 2. Dendrofenograma de B. scarlatina (n=8) marzo de 2014 a octubre 2015. Reserva Biológica Alberto Manuel Brenes, Costa Rica. (cf: caída de follaje, br: brotes foliares, fl: floración, fr: fructificación). 
Figure 2. Dendrofenogram of B. scarlatina $(\mathrm{n}=8)$ March 2014 to October 2015. Alberto Manuel Brenes Biological Reserve, Costa Rica. (cf: leaf abscission, br: budding, fl: flowering, fr: fructification).

El comportamiento fenológico de los individuos 1,2,5 y la media de la población varió entre estos. En cuanto a la caída de follaje, se nota que en junio 2014 fue el mes en que el fenómeno se ausentó en los tres arbustos y en la media. En mayo 2014 hay una tendencia a bajar tanto en las plantas observadas, como en la población estudiada. Por el contrario, el máximo valor en caída de follaje se produjo en febrero 2015 (Figura 3).

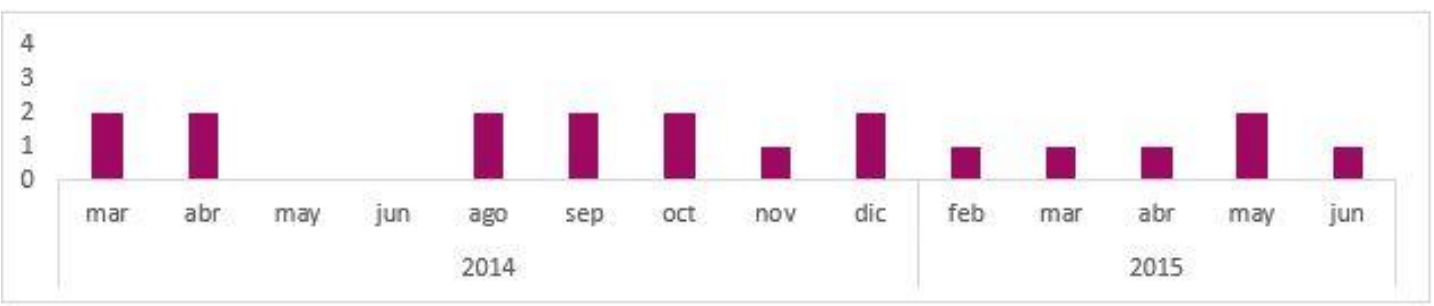

1

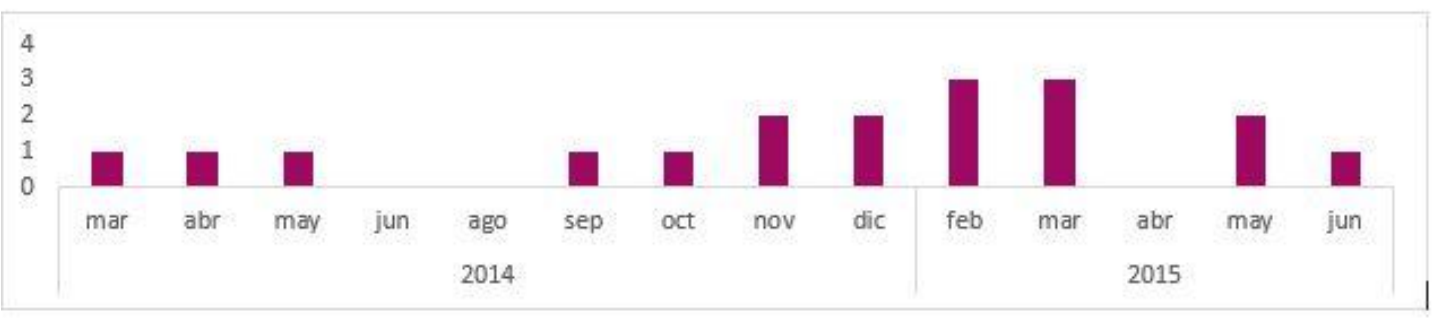

2

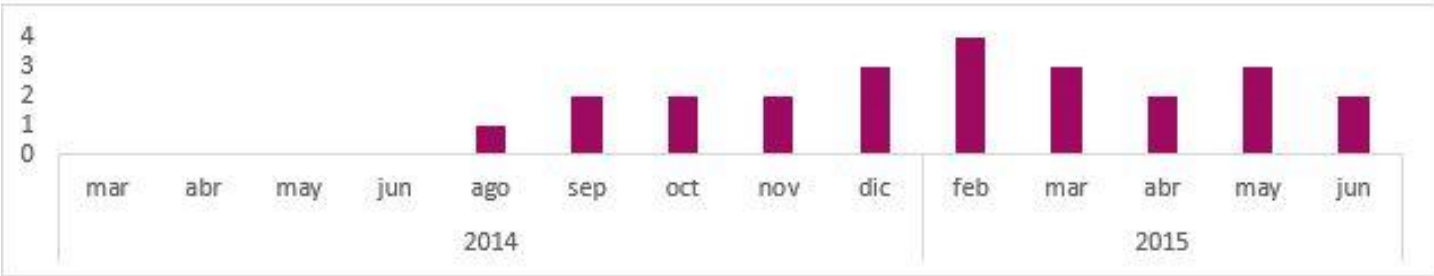

5

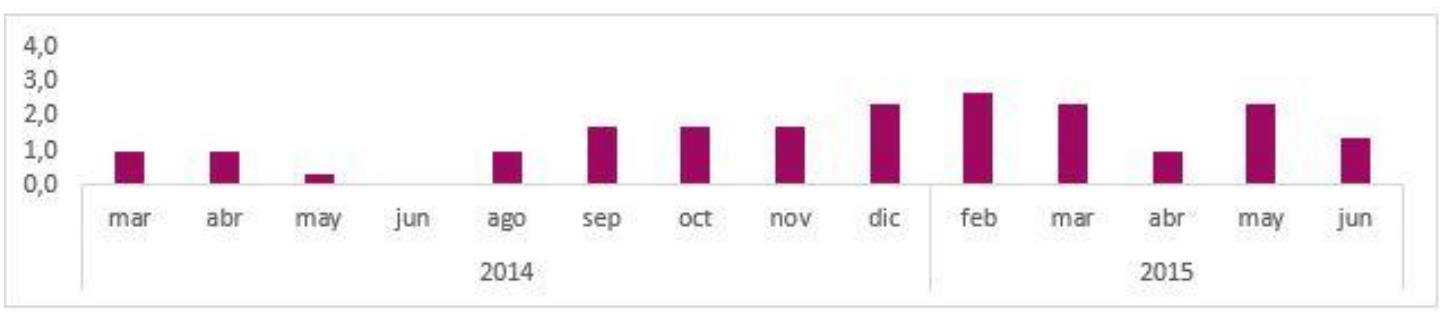

Promedio 
Figura 3. Promedio total $(\mathrm{T})(\mathrm{n}=8)$ de caída de follaje en tres de los ocho árboles estudiados y tomados al azar (1,2,5 de B. scarlatina).

Figure 3. Total average $(\mathrm{T})(\mathrm{n}=8)$ of leaf abscission in three of the eight shrubs studied and taken at random $(1,2,5$ of $B$. scarlatina $)$.

Se aprecia una producción relativamente alta de brotes foliares en el eje 1 y en el 5, contrario a lo que ocurrió con el individuo 2. La ausencia de brotes foliares es escasa, dado que no se observaron en agosto 2014 (individuo 2) y marzo 2014 (individuo 5) (Figura 4).

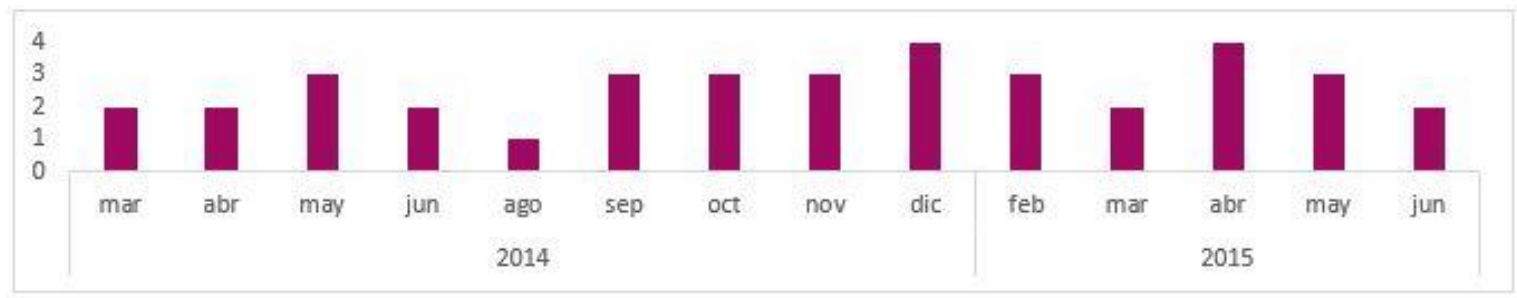

1

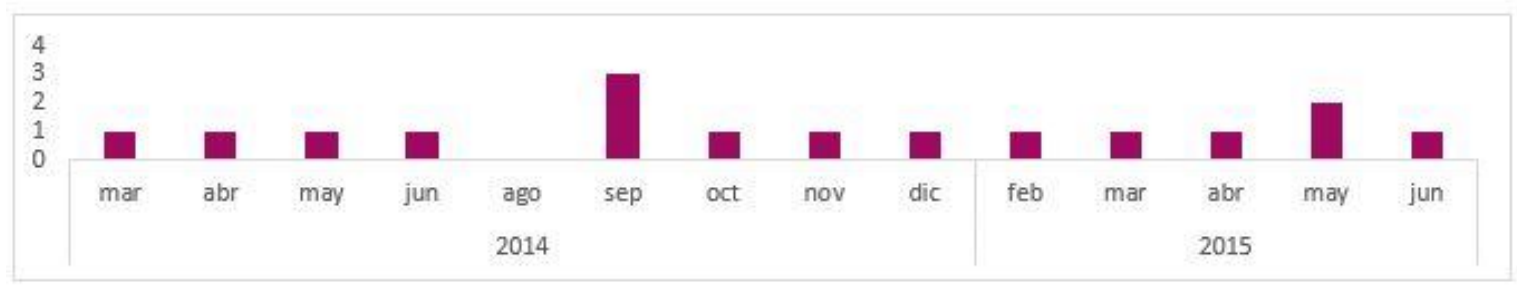

2

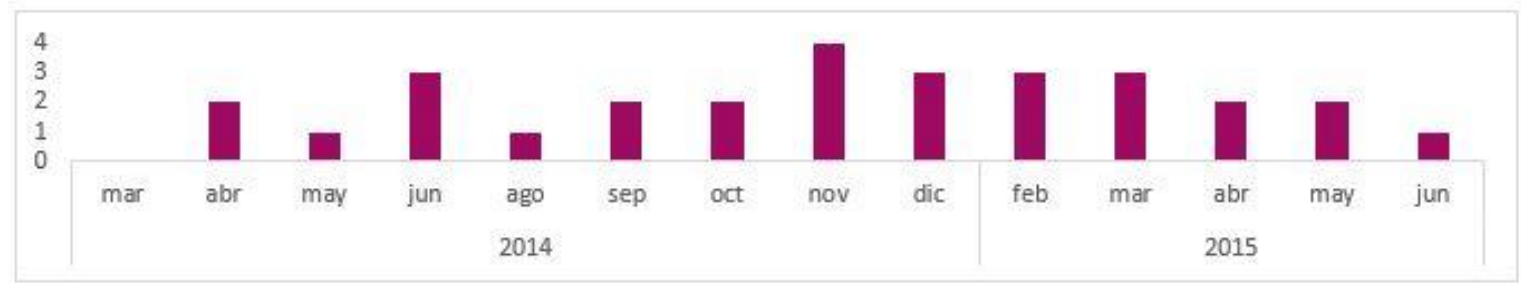

5

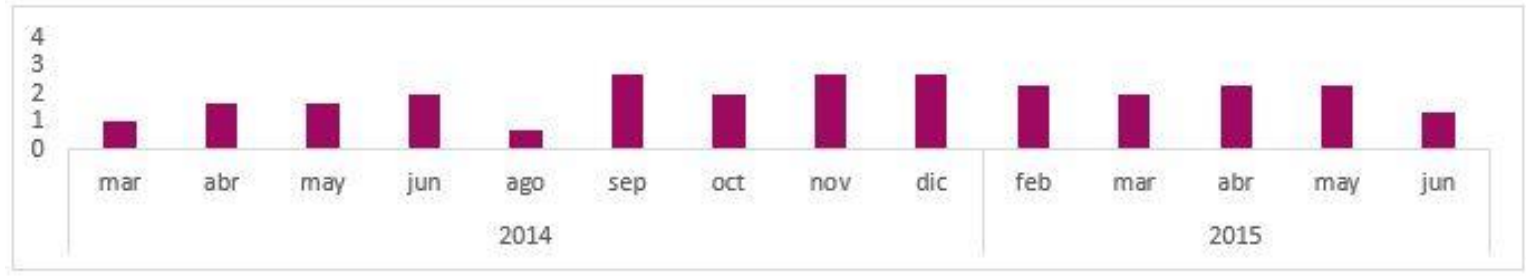

Promedio 
Figura 4. Promedio total $(\mathrm{T})(\mathrm{n}=8)$ de brotes foliares en tres de los ocho arbustos estudiados y tomados al azar (1,2,5 de B. scarlatina).

Figure 4. Total average $(T)(n=8)$ of budding in shrubs of the eight shrubs studied and taken at random (1,2,5 of B. scarlatina).

La floración (Fig. 1Ay 1B), al igual que con los brotes foliares, también se produjo en mayor proporción en el individuo 1, seguido del 5 y, por último, en el 2. En agosto 2014 fue la mayor producción de flores en la muestra y durante el estudio. Esta especie presentó floración durante el 64\% del estudio (Figura 5).

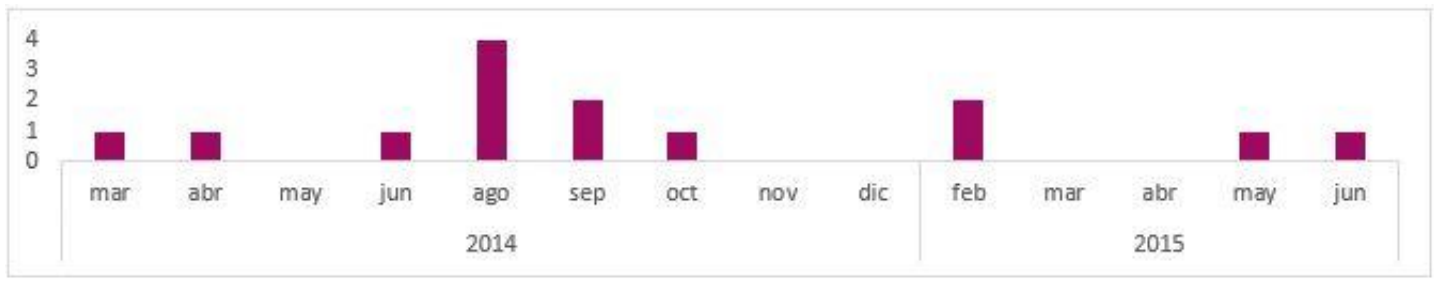

1

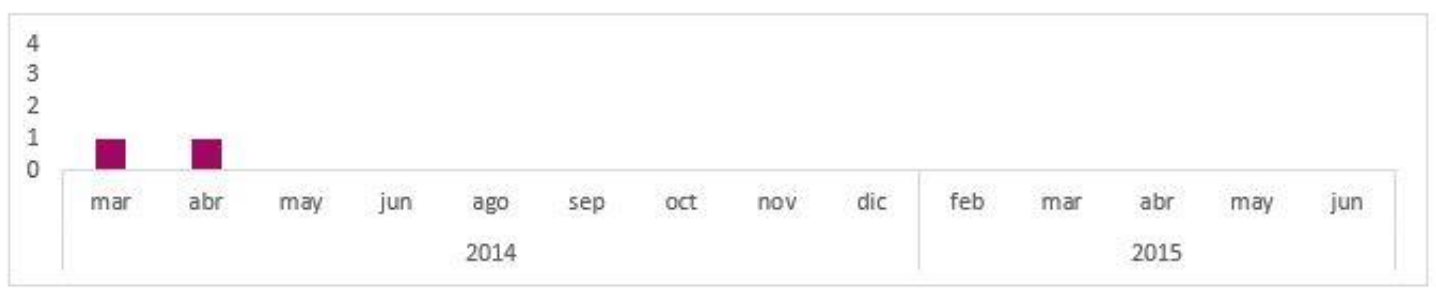

2

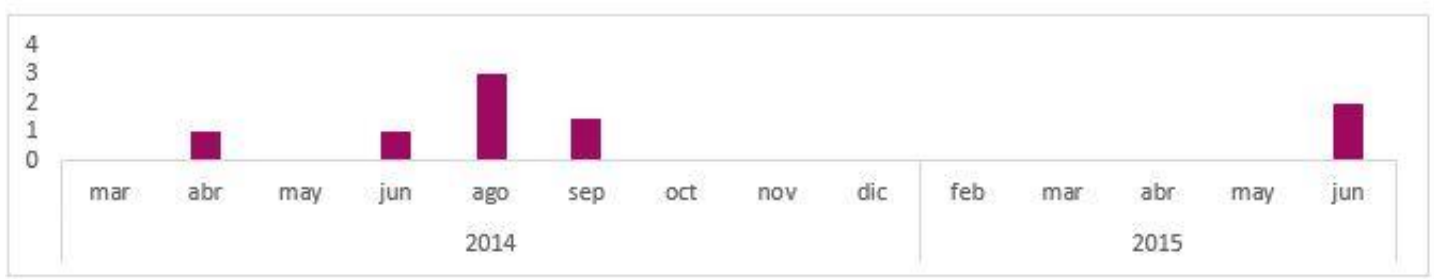

5

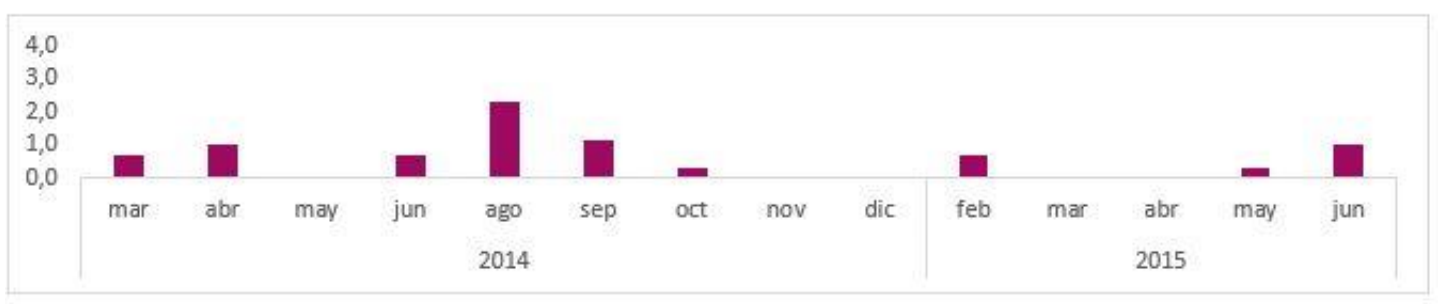

Promedio 
Figura 5. Promedio total $(\mathrm{T})(\mathrm{n}=8)$ de floración en tres de los ocho arbustos estudiados y tomados al azar (1,2,5 de B. scarlatina).

Figure 5. Total average $(\mathrm{T})(\mathrm{n}=8)$ of flowering in three of the eight shrubs studied and taken at random (1,2,5 of B. scarlatina).

B. scarlatina mostró también en el individuo 1 mayor fructificación, seguido del individuo 5 y por último el 2. Al igual que con la floración en el $64 \%$ del estudio se presentó fructificación (Figura 6).

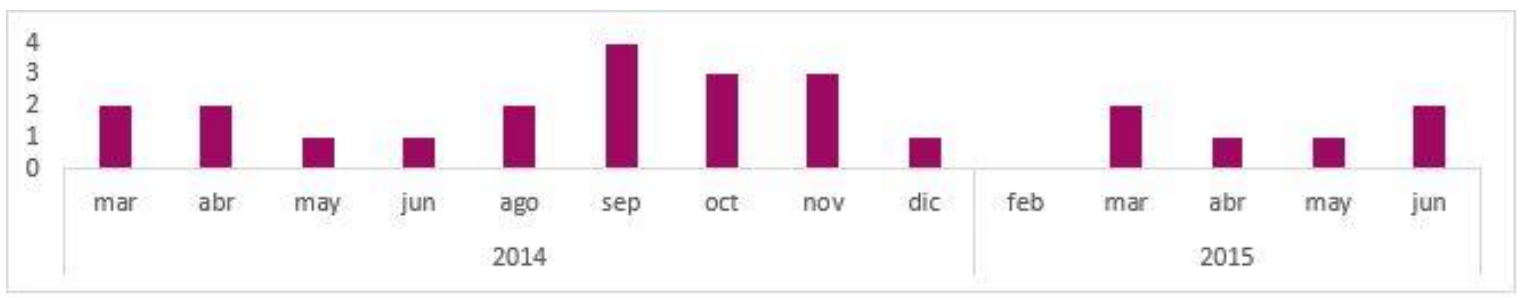

1

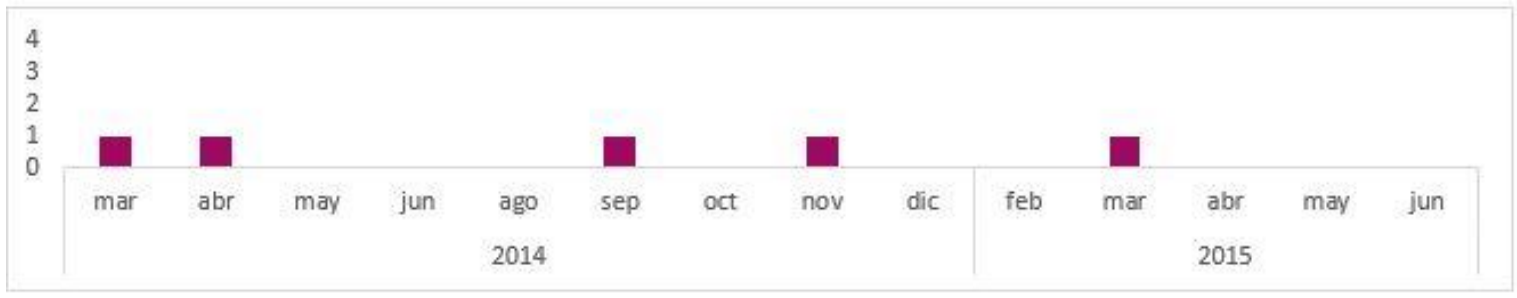

2

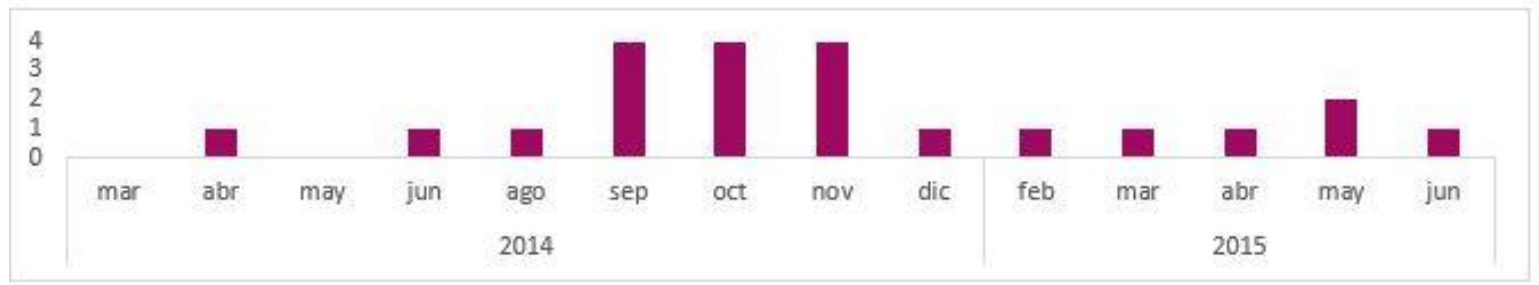

5

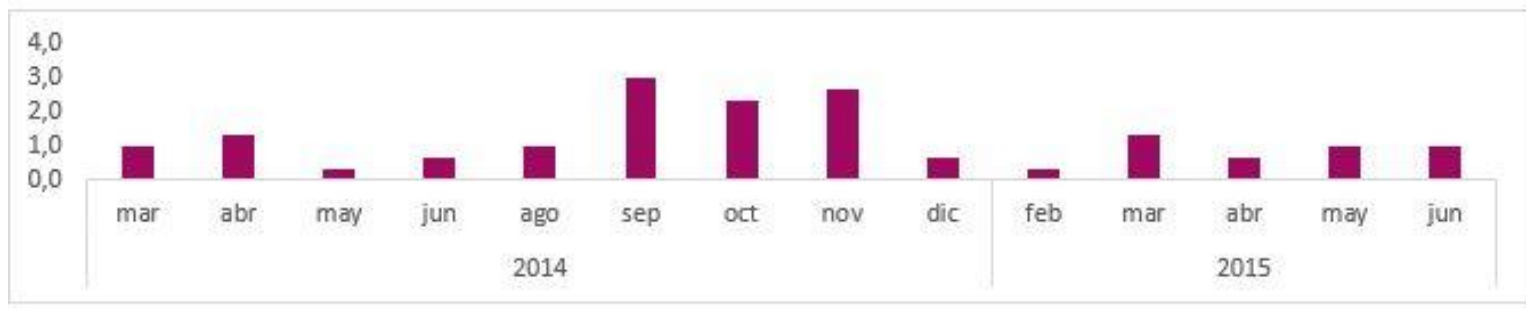

Promedio 
Figura 6. Promedio total $(\mathrm{T})(\mathrm{n}=8)$ de floración en tres de los ocho arbustos estudiados y tomados al azar (1,2,5 de B. scarlatina).

Figure 6. Total average $(\mathrm{T})(\mathrm{n}=8)$ of flowering in three of the eight shrubs studied and taken at random (1,2,5 of B. scarlatina).

La caída de follaje y los brotes fueron inversamente proporcional con la temperatura $(n=12$, $\mathrm{r}=-0.57, \mathrm{p}<0.05)$ y $(\mathrm{n}=12, \mathrm{r}=-0.32, \mathrm{p}<0.05)$ respectivamente. En cuanto a la floración se asoció con la temperatura $(\mathrm{n}=12, \mathrm{r}=0.34, \mathrm{p}<0.05)$ En cambio, la floración de B. scarlatina inversamente asociada con la humedad relativa $(n=12, r=-0.54, \mathrm{p}<0.05)$. Por último, la fructificación se asoció con la precipitación $(\mathrm{n}=12, \mathrm{r}=0.47, \mathrm{p}<0.05)$ (Figura 7).

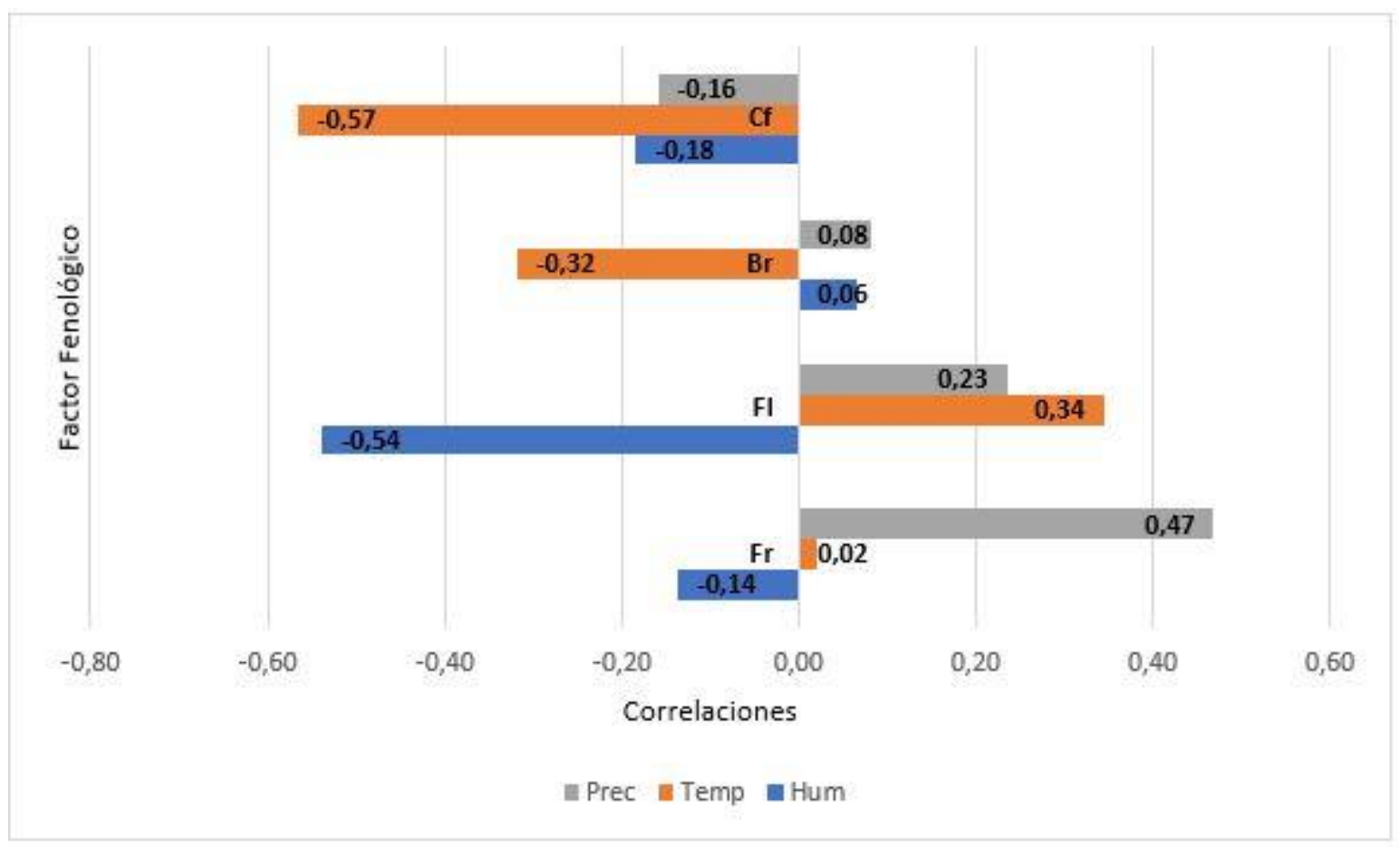

Figura 7. Correlaciones lineales ( $\mathrm{n}=12)$ entre 1: caída de follaje (cf), 2: brotes (br), 3: floración (fl), 4: fructificación (fr) con precipitación (Prec), temperatura (Temp) y humedad relativa (Hum), en B. scarlatina, Reserva Biológica Alberto Manuel Brenes, Costa Rica.

Figure 7. Linear correlations $(\mathrm{n}=12)$ between 1 : leaf abscission $(\mathrm{cf}), 2$ : budding(br), 3: flowering (fl), 4: fructification (fr) with precipitation (Prec), temperature (Temp) and relative humidity (Hum), in B. scarlatina, Alberto Manuel Brenes Biological Reserve, Costa Rica. 


\section{DISCUSIÓN}

La caída de follaje se presentó durante todo el estudio excepto en junio 2014. Esta especie no presentó un patrón fenológico regular, sino que osciló a través del periodo de estudio, esto concuerda con lo dicho por Boyle y Bronstein (2012) los cuales expresan que los diversos patrones fenológicos de las especies fueron relativamente no estacionales a nivel de comunidad y paisaje.

La presencia de brotes foliares se encontró durante todo el estudio, semejante a lo visto por Brenes y Sánchez (2017) en la especie Talauma gloriensis en el mismo sitio. Por otro lado, Brenes y Di Stefano (2001) registraron en Elaeagia uxpanapensis datos distintos, en el que se produjo brotes foliares en periodos de dos y hasta tres y ocho meses.

B. scarlatina produjo flores en nueve de los 14 meses de investigación. Según Newstron, Frankie y Baker (1994) que proponen una clasificación de las plantas basado en patrones de floración, esta especie corresponde a un patrón continuo ya que florece con periodos cortos esporádicos durante el año. Esta característica de patrón continuo de floración hace a $B$. scarlatina una especie muy atractiva a utilizar como planta ornamental.

También se observó que hay un traslape en las fenofases de floración y fructificación en tres momentos del estudio, lo que coincide con Gómez, Hahn y San Martín (2014) quienes explican que se observó cierta sincronía con expresión de todas las fenofases de flores y frutos de Adesmia bijuga Phil (Fabaceae), en un hábitat costero mediterráneo perturbado de Chile Central.

La floración en el individuo 1 al igual que con los brotes foliares, también se produjo en mayor proporción en el individuo 1, seguido del individuo 5 y por último el 2. En agosto 2014 fue la mayor producción de flores en la muestra y durante el estudio. Esta especie presentó floración durante el $64 \%$ del estudio. Este comportamiento fenológico, en cuanto a floración no coincide con lo observado por Singh y Kushwaha (2006), en un bosque tropical deciduo en la India, en el que nueve especies presentaron floración en diferentes meses del año. Por su lado, Cortés-Flores, Cornejo-Tenorio e Ibarra- Manríquez (2011) encontraron en Michoacán, México una alta sincronía intraespecífica en más del $60 \%$ de los taxones estudiados, diferente a lo observado investigado en B. scarlatina en la que no existe una sincronía tan marcada entre los individuos de la especie.

De nuevo se distingue que $B$. scarlatina mostró en el individuo 1 mayor fructificación, seguido del 5 y por último el 2. Al igual que con la floración en el 64\% del estudio se presentó 
fructificación. Cortés-Flores, Cornejo-Tenorio e Ibarra- Manríquez (2011) hallaron que Alnus jorullensis y Quercus obtusata que mostraron una fructificación asincrónica intraespecífica al igual que $B$. scarlatina. En la que se sospecha es una especie zoócora.

Vílchez Chazdon y Alvarado (2008) en un estudio hecho en Sarapiquí, Heredia, Costa Rica, reconocieron que del 38,20\% de las especies supranuales del dosel tienen fructificación continua extensa de un $10.11 \%$, en todo el año hay disposición de frutos como ocurre en esta especie de Melastomataceae estudiada.

La caída de follaje y los brotes fueron inversamente proporcional con la temperatura ( $\mathrm{n}=12$, $\mathrm{r}=-0.57, \mathrm{p}<0.05)$ y $(\mathrm{n}=12, \mathrm{r}=-0.32, \mathrm{p}<0.05)$ respectivamente. En cuanto a la floración se asoció con la temperatura $(\mathrm{n}=12, \mathrm{r}=0.34, \mathrm{p}<0.05)$ tal como lo encontró Pérez, Villalba y Almanza (2013) en Cauca, Colombia con Quercus humboldtii Bonpland el cual también se asoció con la temperatura. En cambio, la floración de B. scarlatina inversamente asociada con la humedad relativa ( $\mathrm{n}=12$, $\mathrm{r}=-$ $0.54, \mathrm{p}<0.05)$.

Por último, la fructificación se asoció con la precipitación ( $\mathrm{n}=12, \mathrm{r}=0.47, \mathrm{p}<0.05)$. Este resultado concuerda con lo encontrado por Brenes y Sánchez (2017) en un estudio realizado en la ReBAMB pero con Talauma gloriensis. Contrario a lo anterior, Cifuentes Moreno y Arango (2013) en un estudio de Euterpe oleracea en la cuenca del río Atrato, Colombia determinaron que los picos máximos de la fructificación de esa especie no se correlacionaron con la precipitación.

Agradecimientos

Se agradece a la Vicerrectoría de Investigación, Coordinación de Investigación de la Sede de Occidente, ambas de la Universidad de Costa Rica, por el financiamiento para realizar la presente investigación. También a Brayan Morera por su apoyo en las labores de campo y a Ronald Sánchez Brenes por su colaboración. 


\section{Referencias}

Almeda, F. 1990.New species and new combinations in Blakea and Topobea (Melastomataceae), with an historical perspective on generic limits in the tribe Blakeae. Proceedings of the California Academy of Sciences Series 4, 46(14): 314.

Almeda, F.2000. A Synopsis of the Genus Blakea (Melastomataceae) in Mexico and Central America. Novon, 10 (4): 299-319.

Azofeifa-Rojas, C. 2015. Album de Especies: Semillas vivas y suelos vivos. Organización para Estudios Tropicales, Estación Las Cruces, San Vito de Coto Brus. Recuperado de: https://ecomaletas.files.wordpress.com/2014/05/albumsemillas-libres_alta.pdf

Borchert R. 1983. Phenology and control of flowering in tropical trees. Biotropica, 15 (2): 81-89.

Boyle, A. y Bronstein, J.2012. Phenology of tropical understory trees: patterns and correlates. Rev.biol.trop 60 (4). Recuperado de: http://www.scielo.sa.cr/scielo.php?script=sci_arttext\&pid=S0034-77442012000400002

Brenes Cambronero, L. 1999. Autoecologia de Elaeagia uxpanapensis D. Lorence (Rubiaceae), en la Reserva Biológica Alberto Manuel Brenes, San Ramón, Costa Rica. Tesis de Maestría. Universidad de Costa Rica, San Pedro de Montes de Oca, San José, Costa Rica. 116 p.

Brenes Cambronero, L. y Di Stefano, J.F. 2001. Comportamiento fenológico del árbol Elaeagia Uxpanapensis (Rubiaceae), en un bosque pluvial premontano de Costa Rica. Rev.biol.trop 49 (34): 989-999.

Brenes Cambronero, L. Sánchez, R. 2017. Fenología de Talauma gloriensis Pittier (Magnolaceae), Reserva Biológica Alberto Manuel Brenes, San Ramón, Alajuela, Costa Rica. Revista Pensamiento Actual, Vol 17(Suplemento $\mathrm{N}^{\circ}$ 1):11-22.

Cifuentes, L., Moreno, F. y Arango, D.A. 2013. Comportamiento fenológico de Euterpe oleracea (Arecaceae) en bosques inundables del Chocó biogeográfico. Revista Mexicana de Biodiversidad 84: 591-599.

Cortés-Flores, J., Cornejo-Tenorio, G. e Ibarra- Manríquez, G. 2011. Fenología reproductiva de las especies arbóreas de un bosque neotropical. Interciencia 36 (8): 608-613. 
Fournier, L. 1974. Un método cuantitativo para la medición de características fenológicas en árboles. Turrialba 24 (4):422-423.

Gómez-Restrepo ML. 2010-2011. Fenología reproductiva de especies forestales nativas presentes en la jurisdicción de CORANTIOQUIA, un paso hacia su conservación. 2 Volúmenes.Medellín: CORANTIOQUIA.

Lieberman D. 1982.Seasonality and phenology in a dry tropical forest in Ghana. Journal of Ecology, 70 (3): 791-806.

Martins, A.B. 2009. Melastomataceae. In: Martins, S.E., Wanderley, M.G.L., Shepherd, G.J., Giulietti, A.M., Melhem, T.S. (eds.) Flora Fanerogâmica do Estado de São Paulo. Instituto de Botânica, São Paulo, vol. 6, pp: 1-168.

Newstrom, L.E., G.W. Frankie, H.G. Baker \& R.K. Colwell. 1994. Diversity of long-term flowering patterns at La Selva, p. 142-160. In L.A. McDade, K.S. Bawa, G.S. Hartshorn \& H.A. Hespenheide (eds.). La Selva: Ecology and Natural History of a Neotropical Rain Forest. University of Chicago, Chicago, Illinois, USA.

Persy, G., Hahn, S. y San Martín, J. 2014. Estructura y fenología reproductiva de una población remanente de Adesmia bijuga Phil., Fabaceae, en un hábitat costero mediterráneo perturbado de Chile central. Gayana bot. 71 (1) 163-166.

Pérez, C.A., Villalba, J.C. y Almanza, M.I. 2013. Fenología del Roble (Quercus humboldtii Bonpland), en Popayán (Cauca, Colombia). Biotecnología en el Sector Agropecuario y Agroindustrial 2: 145-154.

Primack RB (1987) Relationship among f lowers, fruits and seeds. Annu. Rev. Ecol. Syst.18: 409-430.

Rathcke B, Lacey EP (1985) Phenological patterns of terrestrial plants. Annu. Rev. Ecol. Syst. 16:179-214.

Schwartz MD. 2003. Introduction. En: Schwartz MD, editor. Phenology: an integrative environmental science. Dordrecht: Kluwer Academic Publishers. p. 1-7. 
InterSedes, $\mathbf{N}^{\circ}$ 42. Vol XX (2019). ISSN 2215-2458

Singh, K. y Kushwaha, C. 2006. Diversity of Flowering and Fruiting Phenology of Trees in a Tropical Deciduous Forest in India. Ann Bot. 97 (2): 265-276.

Vílchez, B., Chazdon, R. y Alvarado, W. 2008. Fenología reproductiva de las especies del dosel en bosques secundarios y primarios de la región Huetar Norte de Costa Rica y su influencia en la regeneración vegetal. Revista Forestal Kurú 5 (15): 1-18 